\title{
Research on fixed attack angle attack angle and height flying of hypersonic aircraft
}

\author{
Guangbin $\mathrm{Wu}^{1}$ Heng $\mathrm{Li}^{2}$ and Junwei $\mathrm{Lei}^{1, \mathrm{a}}$ \\ ${ }^{1}$ Department of control engineering, Naval aeronautical and astronautical University \\ ${ }^{1}$ Receiving and training center of new equipment, Naval aeronautical and astronautical University \\ Yanti, 264001 \\ aleijunwei@126.com
}

Keywords: hypersonic aircraft;fixed attack angle; fixed height; numerical simulation

\begin{abstract}
Attack angle is a most important factor that affect the stability of aircraft no matter for supersonic type or hypersonic type aircraft. And height is another factor that will affect the force and moment of the whole dynamic system by change the density of air and finally it will also affect the stability of the whole system. So in this paper a kind of virtual flying with fixed attack angle and height is proposed to testify the rightness of the hypersonic aircraft model and air coefficient. It is a new and meaningful model testifying method for complex flying systems.
\end{abstract}

\section{Introduction}

The fixed height flying test is an usual effective measure for traditional non- hypersonic aircraft, because it can avoid the changes of the air density caused by the changes of the height ${ }^{[1,2]}$. Then pressure varies, lead to the changes of the stress on aircraft. We can verify the aircraft's dynamic character accurately in the fixed height nearby through the fixed height flying test ${ }^{[4-7]}$. The change of aerodynamic force caused by the change of height is vital part of the interferences 。 But for hypersonic aircraft, its influence is not exceeding the engine and the changes of the elastics, ${ }^{\text {[8,9] }}$. So this paper does the fixed height simulation based on the fixed attack angle. The results indicate the characteristic of hypersonic aircraft mentioned above.

\section{Model Description}

Considering the elastic shape structure, a kind of pitch channel hypersonic aircraft model built according to Lagrange equation is released by USA air force as followed:

$$
\begin{gathered}
\dot{V}=\frac{T \cos \alpha-D}{m}-g \sin \gamma \\
T=\bar{q} s\left(C_{T \phi} \phi+C_{T}+C_{T}^{\eta} \eta\right) \\
C_{T}^{\eta} \eta=6.783 * 10^{-2} \eta_{1}+3.226 * 10^{-2} \eta_{2}+8.635 * 10^{-2} \eta_{3} \\
D=\bar{q} S C_{D} \\
\dot{\phi}=-2 \varsigma \omega_{n} \phi-\omega_{n}^{2} \phi+\omega_{n}^{2} \phi_{c}, \omega_{n}=5, \varsigma=0.7 \\
N_{i}^{\eta}=\left[N_{i}^{\eta_{1}}, 0, N_{i}^{\eta_{2}}, 0, N_{i}^{\eta_{3}}, 0\right] \\
\left.C_{D}=C_{D}^{\alpha^{2}} \alpha^{2}+C_{D}^{\alpha} \alpha+C_{D}^{\delta_{e}^{2}} \delta_{e}^{2}+C_{D}^{\delta_{e}} \delta_{e}+C_{D}^{0}+C_{D}^{\eta_{2}}, 0, C_{j}^{\eta_{3}}, 0\right], j=T, M, L, D \\
=5.8224 \alpha^{2}-0.0045315 \alpha+0.81993 \delta_{e}^{2}+0.00027699 \delta_{e} \delta_{c} \\
+0.01013+0.7186 \delta_{c}^{2}+0.00038522 \delta_{c}+C_{D}^{\eta} \eta+5.346 * 10^{-2} \eta_{1}+5.346 * 10^{-2} \eta_{2}+0.543 \eta_{3}
\end{gathered}
$$




$$
\begin{gathered}
C_{T \phi}=C_{T \phi}^{\alpha^{3}} \alpha^{3}+C_{T \phi}^{\alpha^{2}} \alpha^{2}+C_{T \phi}^{\alpha} \alpha+C_{T \phi}^{0}=15.436 \alpha^{3}+6.2324 \alpha^{2}+7,9436 \alpha+0.5887 \\
C_{T}=C_{T}^{\alpha^{3}} \alpha^{3}+C_{T}^{\alpha^{2}} \alpha^{2}+C_{T}^{\alpha} \alpha+C_{T}^{0}=10.936 \alpha^{3}+8.438 \alpha^{2}+6.564 \alpha+6.960 \\
\dot{\gamma}=\frac{L+T \sin \alpha}{m V}-\frac{g \cos \gamma}{V} \\
L=\bar{q} S C_{L}, \\
C_{L}=C_{L}^{\alpha} \alpha+C_{L}^{\delta_{e}} \delta_{e}+C_{L}^{\delta_{c}} \delta_{c}+C_{L}^{0}+C_{L}^{\eta} \eta \quad \\
=4.6773 \alpha+0.76224 \delta_{e}+0.7408 \delta_{c}-0.0018714+4.249 * 10^{-2} \eta_{1}+0.01104 * \eta_{2}+1.785^{*} 10^{-2} \eta_{3} \\
\dot{\alpha}=q-\dot{\gamma} \\
\dot{q}=\frac{M}{I} \\
M=z_{T} T+\bar{q} S \bar{c} C_{M} \\
C_{M}=C_{M}^{\alpha^{2}} \alpha^{2}+C_{M}^{\alpha} \alpha+C_{M}^{\delta_{e}} \delta_{e}+C_{M}^{\delta_{c}} \delta_{c}+C_{M}^{0}+2.569 * 10^{-2} \eta_{1}-0.3746 \eta_{2}+5.449 * 10^{-2} \eta_{3} \\
=6.2926 \alpha^{2}+2.1335 \alpha+0.1986 \delta_{e}+0.23048 \delta_{c}+0.18979+C_{M}^{\eta} \eta \\
\dot{h}=V \sin \gamma \\
\ddot{\eta}_{i}=-2 \varepsilon_{m} \omega_{m i} \dot{\eta}_{i}-\omega_{m i}^{2} \eta_{i}+N_{i}, \quad N_{i}=\bar{q} S C_{N i} \\
C_{N_{i}}=C_{N_{i}}^{\alpha^{2}} \alpha^{2}+C_{N_{i}}^{\alpha} \alpha+C_{N_{i}}^{\delta_{e}} \delta_{e}+C_{N_{i}}^{\delta_{c}} \delta_{c}+C_{N_{i}}^{0}+N_{i}^{\eta} \eta
\end{gathered}
$$

And $V$ is speed, $\gamma$ is the speed angle, $\alpha$ is attack angle, $Q$ is the attitude angle speed, $h$ is the height. $\phi$ is the oil supplying factor, $\delta_{c}$ is the duck wing and $\delta_{e}$ is the lift rudder.

\section{Simulation settings of free flying}

In order to testify the rightness of the model of hypersonic aircraft, choose a initial height as $h_{0}=85000 * 0.3048$, initial speed as $V_{0}=7846 * 0.3048$, initial attack angle as $\alpha_{0}=0.0174$, and other initial state as: $\gamma_{0}=0 \quad, \quad q_{0}=0 \quad, \quad \eta_{10}=0.4588 * 0.3048 * 14.59 \quad, \quad \eta_{20}=-0.08726 * 0.3048 * 14.59$, $\eta_{30}=-0.03671 * 0.3048 * 14.59$, and set the oil supplying law as constant as $\phi=\phi_{c}=0.1$ and choose the control as $\delta_{c}=0$, then the fixed attack angle and height flying test can be done by numerical simulation to make $\dot{\alpha}=0, \quad \dot{h}=0$, and simulation time is set as 0.5 second.

\section{Numerical Simulation and result analysis}

With above model and air coefficients and set initial condition as above paragraph and write a program with $\mathrm{m}$ language in Matlab software, then the simulation can be done and simulation results can be shown as following figures. 


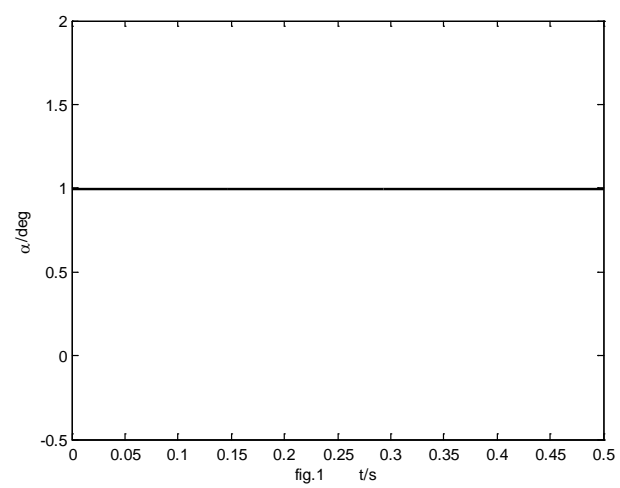

Fig 1 The curve of attack angle

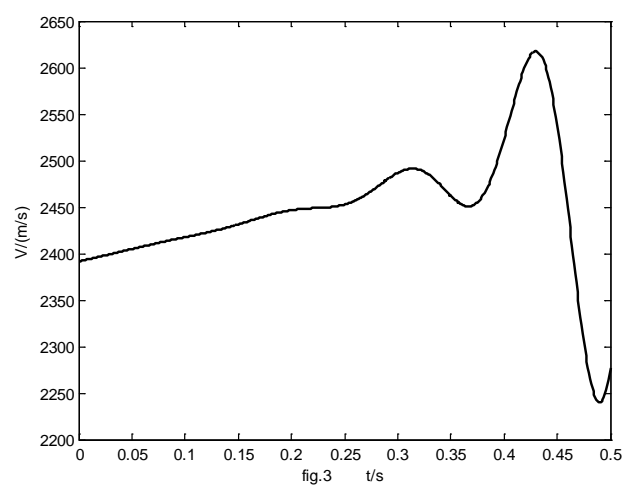

Fig 3 The curve of speed

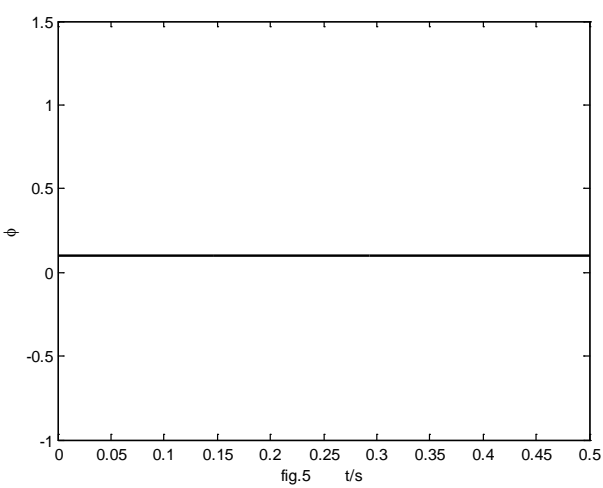

Fig 5 The curve of oil supplying factor

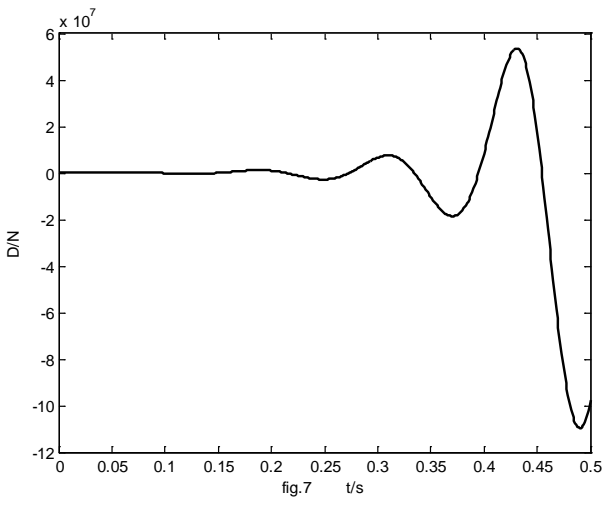

Fig 7 The curve of resistance

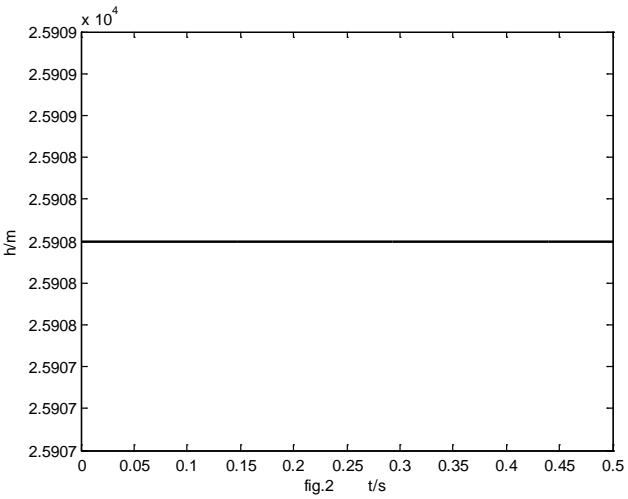

Fig 2 The curve of the height

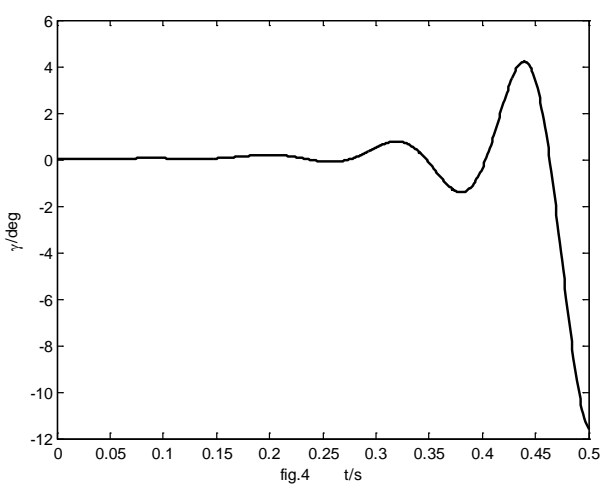

Fig 4 The curve of speed angle

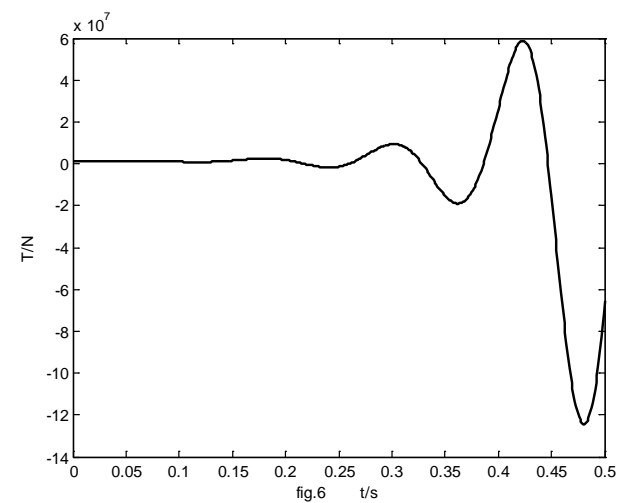

Fig 6 The curve of thrust

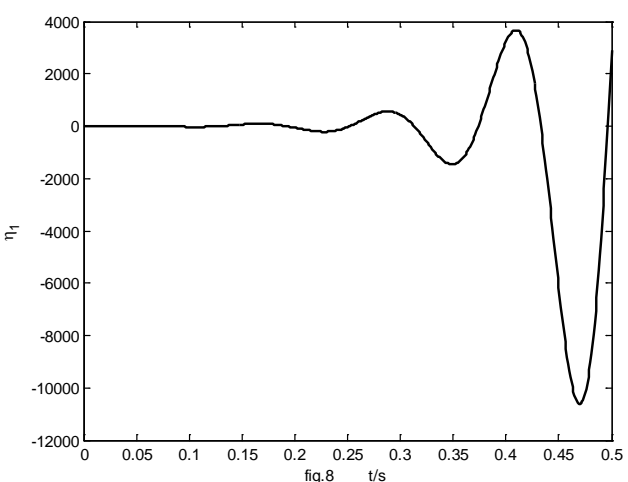

Fig 8 The state of first elastic shape 


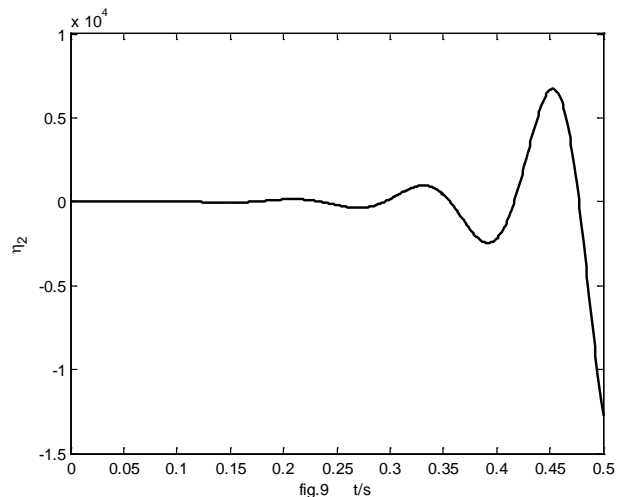

Fig 9 The state of second elastic shape

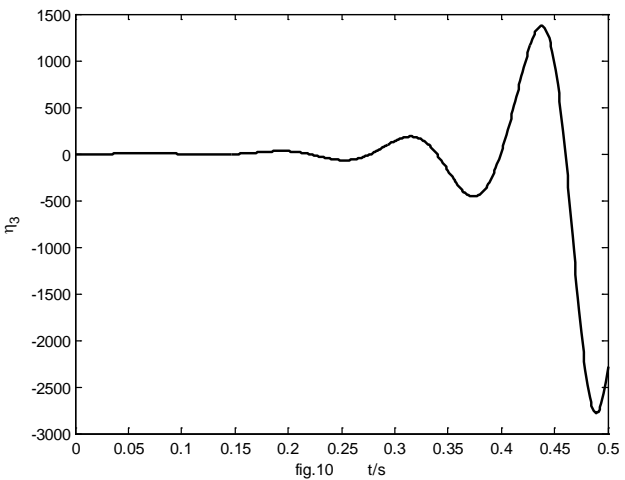

Fig 10 The state of third elastic shape

The trajectory dip angle also disperses on the fixed attack angle and height flying at $0.5 \mathrm{~s}$. It caused by dispersion of elastic deformation of projectile bodies, but the low speed missile does not emerge this situation. Height and dip angle of trajectory all disperse slowly in the fixed attack angle slow speed missile flying. But the variety of elastic form of projectile bodies is not obvious 。 It indicates elastic form is affected by the other factors on the fixed attack angle flying, this fits the mathematic model well.

The fixed attack angle and height flying of hypersonic aircraft is faster than the dispersion of posture of aircraft and elastic form. So we can see posture of aircraft and elastic form of hypersonic aircraft have the functions as the auto-stability and self-calibration。But this function will be vanished, if the attack angle is set as constant. In some situations, the shaking of attack angle has a beneficial effect to the system, because elastic form exists .

\section{Conclusion}

The fixed attack angle and height flying of hypersonic aircraft is stability basically on $0.5 \mathrm{~s}$ through the curves above. So the model of aircraft can be verified stability basically, to accomplish verifying the model preliminarily. But elastic form of engine body of hypersonic aircraft disperses quickly, it causes the stress increasing rapidly and then the posture disperses fast. It has the differences in substance with the traditional hypersonic aircraft. The traditional fixed attack angle hypersonic aircraft and subsonic aircraft can fly stably with the posture and height keeping in the certain range working above 2s. At the same time, the influence of the changing of the elastic form for the system's stability is more intense than the height for the interference in short time in the dynamic process of the hypersonic aircraft.

\section{References}

[1]Walton J T, Ohio C. Performance Sensitivity of Hypersonic Vehicles to Changes in Angle of Attack and Dynamic Pressure[C]//25th Joint Propulsion Conference. Monterey, CA: AIAA press, 1989:1-11.

[2]Stich R, Sachs G. Path-attitude decoupling problems of aerospace craft[C]//AIAA Guidance, Navigation, and Control Conference and Exhibit. Hilton, 2007:25-28.

[3]Sigthorsson D O, Jankovsky P. Output feedback control and sensor placement for a hypersonic vehicle model[C]//AIAA Guidance, Navigation, and Control Conference and Exhibit. Hilton, 2007:20-23

[4]Sigthorsson D O, Jankovsk P, Serrani A. Robust linear output feedback control of an air-breathing hypersonic vehicle[J]. Journal of Guidance, Control and Dynamics, 2008, 31(4):1052-1066

[5]Bolender M A, Doman D B. Nonlinear robust/adaptive controller design for an air-breathing 
hypersonic vehicle model[C]//AIAA Guidance, Navigation, and Control Conference and Exhibit. Hilton, 2007:20-23

[6]Bolender M A, Doman D B. Nonlinear robust adaptive control of flexible air-breathing hypersonic vehicles[J]. Journal of Guidance, Control and Dynamics, 2009, 32(2):739-746

[7]Bolender M A, Doman D B. Combined reference governor and anti-windup design for constrained hypersonic vehicles models[C]//AIAA Guidance, Navigation, and Control Conference and Exhibit. Illinois, 2009:10-13

[8]Heppenheimer T A. Facing the heat barrier: A history of hypersonic[J]. Journal of Spacecraft and Rocket, 2007, 25(7):25-32

[9]Bolender M A, Doman D B. Nonlinear longitudinal dynamical model of an air-breathing hypersonic vehicle[J]. Journal Spacecraft and Rockets, 2007, 44(2): 374-386 